Draft version November 8, 2018

Preprint typeset using $\mathrm{LAT}_{\mathrm{E}} \mathrm{X}$ style emulateapj v. 5/2/11

\title{
A CORRELATION BETWEEN THE ECLIPSE DEPTHS OF KEPLER GAS GIANT CANDIDATES AND THE METALLICITIES OF THEIR PARENT STARS
}

\author{
Sarah E. Dodson-Robinson \\ Astronomy Department, University of Texas at Austin, 1 University Station C1400, Austin, TX 78712 USA \\ Draft version November 8, 2018
}

\begin{abstract}
Previous studies of the interior structure of transiting exoplanets have shown that the heavy element content of gas giants increases with host star metallicity. Since metal-poor planets are less dense and have larger radii than metal-rich planets of the same mass, one might expect that metal-poor stars host a higher proportion of gas giants with large radii than metal-rich stars. Here I present evidence for a negative correlation at the $2.3 \sigma$ level between eclipse depth and stellar metallicity in the Kepler gas giant candidates. Based on Kendall's $\tau$ statistics, the probability that eclipse depth depends on star metallicity is 0.981 . The correlation is consistent with planets orbiting low-metallicity stars being, on average, larger in comparison with their host stars than planets orbiting metal-rich stars. Furthermore, since metal-rich stars have smaller radii than metal-poor stars of the same mass and age, a uniform population of planets should show a rise in median eclipse depth with $[\mathrm{M} / \mathrm{H}]$. The fact that I find the opposite trend indicates that substantial changes in gas giant interior structure must accompany increasing $[\mathrm{M} / \mathrm{H}]$. I investigate whether the known scarcity of giant planets orbiting low-mass stars could masquerade as an eclipse depth-metallicity correlation, given the degeneracy between metallicity and temperature for cool stars in the Kepler Input Catalog. While the eclise depth-metallicity correlation is not yet on firm statistical footing and will require spectroscopic $[\mathrm{Fe} / \mathrm{H}]$ measurements for validation, it is an intriguing window into how the interior structure of planets and even the planet formation mechanism may be changing with Galactic chemical evolution.

Subject headings: Stars: planetary systems - Planets and satellites: formation - Planets and satellites: fundamental parameters - Planets and satellites: composition
\end{abstract}

\section{INTRODUCTION}

Since 1958, astronomers have known that Jupiter and Saturn are smaller than they would be if they were made of pure hydrogen and helium (Demarcus 1958). Even though planetary radii depend on complex equations of state, the simple principle that metal-rich planets are more dense than their metal-poor counterparts of the same mass holds for a wide range of planetary radii (e.g. Fortney \& Nettelmann 2011). Since the heavy-element content of transiting gas giants increases with host star metallicity (Guillot et al. 2006; Miller \& Fortney 2011), one may make a simple prediction: the gas giants orbiting metal-poor stars should have higher radii, on average, than the gas giants orbiting metal-rich stars. Just as the planet detection rate depends on star metallicity (Gonzalez 1998; Santos et al. 2004; Fischer \& Valenti 2005), the typical giant planet interior structure also should change according to the metal content of the host star.

Before the advent of the Kepler mission, there was no large, uniform sample of gas giants available to search for a relationship between stellar metallicity and gas giant size. The surveys of transiting gas giants were heterogeneous and mostly insensitive to planets smaller than $\sim 0.7 R_{\text {Jup }}$ (0.7 Jupiter radii). One notable exception was GJ $436 \mathrm{~b}$ at $0.365 R_{\text {Jup }}$, which orbits an M2.5 star of radius $0.464 R_{\odot}$ (Gillon et al. 2007b). The $K e$ pler spacecraft, launched in March of 2009, is monitoring about 150,000 mostly Solar-type stars, primarily searching for transits of Earthlike planets in the habitable zone

sdr@astro.as.utexas.edu
(Batalha et al. 2010). Yet the Kepler mission is also extraordinarily useful for studies of gas giants, for the first time providing a sample of candidate giant planet hosts observed under uniform conditions and with the sensitivity to detect all of the Neptune-like objects of $\sim 0.3 R_{\text {Jup }}$ and below.

Here, then, is the opportunity to investigate the dependence of planetary interior structure on host star metallicity. Of course, one expects to see gas giants with a range of sizes orbiting all types of Population 1 stars - after all, Jupiter and Neptune orbit the same star but have radii that differ by almost a factor of three. However, the ensemble of planet radii should show some dependence on stellar metallicity if the heavy element contents of planets and their parent stars are truly connected, as suggested by Guillot et al. (2006) and Miller \& Fortney (2011). Likewise, since the Kepler mission is surveying primarily similar, Sunlike stars, one also expects a negative correlation between gas giant eclipse depth and star metallicity. In this article I present the first evidence that such a trend exists - though at this time the eclipse depth-metallicity trend is on tenuous statistical footing and will require follow-up spectroscopic observations for verification.

This article is organized as follows: In $\$ 2 \mathrm{I}$ outline my gas giant selection criteria. In $₫ 3 \mathrm{I}$ present statistical support for a negative correlation between eclipse depth and star metallicity. I discuss possible selection biases that could masquerade as an eclipse depth-metallicity trend in 84. Finally, I examine physical mechanisms besides the simple density effect that could lead to an eclipse depthmetallicity correlation and speculate about the implica- 
tions for planet formation theory in $\$ 5$.

\section{SAMPLE SELECTION}

In February 2011, the Kepler team released data for 1235 planet candidates (Borucki et al. 2011a). While the planet/star radius ratios $R_{p} / R_{*}$ were directly measured from eclipse depths, the values of $R_{*}$ were based on $\log (g)$ measurements from the Kepler Input Cata$\log$ (KIC; Brown et al. 2011). The KIC stellar parameters were computed using photometry in the Sloan $u$, $g, r, i, z$ filters, the intermediate-bandwidth $D 51$ filter, and the 2MASS $J H K$ filters (Skrutskie et al. 2006). Brown et al. (2011) quote a 0.4-dex error bar on $\log (g)$, which translates into a $+58 \% /-37 \%$ radius error for a planet transiting a Sunlike star. Hotter, early- to midF stars have higher $\log (g)$ errors, while subgiants tend to have systematically high $\log (g)$ estimates. Furthermore, Chaplin et al. (2010) performed a detailed asteroseismic follow-up study of three Sunlike stars and found that the KIC $T_{\text {eff }}$ estimates were systematically low. Pinsonneault et al. (2012) also found that temperatures derived from KIC griz photometry were systematically lower than temperatures computed by applying the infrared flux method (Casagrande et al. 2010) to 2MASS photometry (Skrutskie et al. 2006). The problems with $\log (g)$ for hot stars may therefore apply to stars that have apparently Sunlike temperatures according to the KIC. Quoted planet candidate radii from the Kepler data release are subject to significant uncertainties.

However, the Kepler planet candidate radii can still be used to identify a set of likely gaseous, giant planet candidates. Here I define giant planets as objects with bulk density $\rho \lesssim 1.5 \mathrm{~g} \mathrm{~cm}^{-3}$, for which $\sim 50 \%$ or more of the planetary volume is due to the $\mathrm{H} / \mathrm{He}$ atmosphere. According to the planetary interior models of Helled et al. (2011), this volume-based definition of giant planet would include Uranus and Neptune, which are about $65 \% \mathrm{H} / \mathrm{He}$ by volume. Rogers \& Seager (2010) show that HAT-P-11b (Bakos et al. 2010) and GJ 436b (Gillon et al. 2007a; Torres 2007) must also have gas mass and volume fractions similar to Uranus and Neptune. HAT-P-11b, GJ 436b, Uranus and Neptune have radii of $4.7 R_{\oplus}, 4.2 R_{\oplus}, 4.1 R_{\oplus}$, and $4.0 R_{\oplus}$, respectively, suggesting that gas giants in general have radii $R_{p} \geq 4 R_{\oplus}$. To be conservative, I follow Schlaufman \& Laughlin (2011) and set the minimum planet candidate radius for this study at $R_{p}=5.0 R_{\oplus}$. For a $5 R_{\oplus}$ candidate with a $+58 \%$ measurement error on planet size, the true size would be $3.2 R_{\oplus}$, still within the size range where a gaseous atmosphere is likely (Rogers \& Seager 2010).

The basic sample consists (1) of candidate giant planets with radius $R_{p} \geq 5.0 R_{\oplus}$ that (2) also have metallicity measurements from the KIC. Of the candidates that satisfy criteria (1) and (2), 28 have estimated sizes larger than the "styrofoam planet" Kepler-7b $\left(R_{p}=18.1 R_{\oplus}\right.$; Latham et al. 2010), the lowest-density transiting planet yet known. Given that theoretical mass-radius relations predict that Jupiter-mass $\left(M_{\text {Jup }}\right)$ planets, $20 M_{\text {Jup }}$ brown dwarfs and $100 M_{\text {Jun }}$ stars have radii that vary by less than 5\% (Chabrier et al. 2009), it is unfortunately very easy for eclipsing low-mass companion stars to masquerade as giant planets. I therefore carefully scrutinized the light curves of the largest planet candidates for signs of (a) planet radius mismeasurement due to $\mathrm{V}$-shaped eclipses, (b) odd/even eclipse depth differences that indicate eclipsing binaries (either in the target itself or a background object), and (c) pulsations that suggest the $\delta$ Scuti-like variability of early- to mid-F stars, for which the $\log (g)$ uncertainties are high.

Among the 23 candidates with metallicities from KIC and $R_{p} \geq 18.1 R_{\oplus}$, I find 7 objects for which the reported ratio $R_{p} / R_{*}$ was mis-fit by the Kepler data analysis pipeline. All such light curves show V-shaped eclipses rather than flat-bottomed eclipses, which drives the fitting algorithm toward artificially low impact parameters and high values of $R_{p} / R_{*}$ (B. Cochran, private communication). Two objects, KIC 5649956 and KIC 1432214 , show the odd/even differences in eclipse depth that indicate an eclipsing binary system. KIC 5649956 pulsates at the $3-4 \%$ level, making the odd/even effect difficult to identify. KIC 6470149 and KIC 7449844, which do not have odd/even eclipse patterns, have been shown to host eclipsing $\mathrm{M}$ dwarfs by follow-up RV observations taken since the release of the Kepler planet candidate list. Meanwhile, follow-up spectra of KIC 5356593 have raised its $\log g$ value from the 3.8 dex listed in the KIC to 4.5 dex, correspondingly shrinking the computed planet radius from $35.5 R_{\oplus}$ to $13-14 R_{\oplus}$ (B. Cochran, private communication).

Furthering the list of suspicious objects, six other stars have no obvious mismeasurement of eclipse depth, but their eclipses are V-shaped, which is more consistent with a companion star than a planet. One star, KIC 10616571, pulsates at the $3 \%$ level on three frequencies, while one or two small peaks occur at the minimum of each eclipse, suggesting a hierarchical triple system. Finally, five stars that do not have $\mathrm{V}$-shaped eclipses or odd/even effects pulsate with similar amplitude to the transit signal. One such pulsator, KIC 11818800, will have its planet candidate radius revised from $36 R_{\oplus}$ to $9.77 R_{\oplus}$ in the upcoming re-release of the Kepler planet candidate list (B. Cochran, private communication). The preponderance of problems among the largest planet candidates suggest that one should exercise caution when selecting a sample of inflated giant planets.

In a planet candidate list ordered according to $R_{p}$, I found that pulsations, odd/even effects and mismeasured and/or V-shaped eclipses accounted for fully $100 \%$ of the objects with $R_{p} \gtrsim 22 R_{\oplus}$. Below $20 R_{\oplus}$, light curves that have no obvious problems begin to dominate. The confirmed planet Kepler-12b has $R_{p}=18.9 R_{\oplus}$ (Fortney et al. 2011) and a similar density to Kepler-7b, validating the idea that such inflated planets can exist. I therefore set an upper limit of $20 R_{\oplus}$ to the planet candidates in this study. The basic sample consists of 213 giant planet candidates with $5.0 R_{\oplus}<R_{p}<20 R_{\oplus}$ whose host stars also have metallicities from the Kepler Input Catalog (KIC). All planet candidates used in this study are listed in Table 1.

While Morton \& Johnson (2011) argue for a false positive rate of $5 \%-10 \%$ among the Kepler planet candidates, Borucki et al. (2011a) are more cautious and quote $>80 \%$ reliability for rank 2 KOIs (Kepler Objects of Interest) and $>60 \%$ reliability for rank 3 and 4 KOIs. Despite the scrutiny of planet candidates, there is therefore still the possibility that the sample is contaminated by as much as $40 \%$ eclipsing binaries, background eclipsing 
binaries and hierarchical triples. I discuss the possible consequences of such contamination in 84

\section{STATISTICAL EVIDENCE FOR DEEP ECLIPSES OF LOW-METALLICITY STARS}

With a sample of gas giant candidates in hand, I now present the first statistical evidence that Galactic chemical evolution affects the internal structure of giant planets as well as the frequency with which they form. Figure 1 shows $R_{p} / R_{*}$ as a function of host star $[\mathrm{M} / \mathrm{H}]$ for the stars in my sample. Planet candidates are color-coded according to the irradiation they receive from their host stars, which I discuss further in \$5. Although I used the Kepler estimates of $R_{p}$ to select my sample of gas giant candidates (see 92 for selection criteria), for the purposes of statistical analysis I have cast the trend in terms of $R_{p} / R_{*}$ rather than $R_{p}$ because planet radii tend to change substantially between inclusion in the Kepler planet candidate list and eventual confirmation and publication. $R_{p} / R_{*}$, by contrast, is a simple observable whose accuracy I have verified by examining the light curves of the largest planet candidates. The question I am asking is - are Kepler candidate giant planets orbiting low-metallicity stars larger in comparison to their host stars than candidate planets orbiting highmetallicity stars?

Figure 1 shows eclipse depth as a function of $[\mathrm{M} / \mathrm{H}]$ for the candidate giant planet hosts in the selected sample. Scanning from left to right, notice the knot of small planet candidates with $0.05 \lesssim R_{p} / R_{*} \lesssim 0.13$ that appears at $[\mathrm{M} / \mathrm{H}] \gtrsim-0.2$ without a commensurate increase in large planet candidates with $R_{p} / R_{*} \gtrsim 0.13$. To quantify the trend in decreasing eclipse depth with metallicity, I have computed a running median (Härdle \& Steiger 1995) with a 21-point window (solid black) and a local polynomial regression (Cleveland et al. 1992) with span 0.75 and degree 1 (dash-dot black). Notice the decreasing nature of both manifestations of the trend in the range $-0.1 \lesssim[\mathrm{M} / \mathrm{H}] \lesssim 0.3$. While planets of all radii can appear around stars of all metallicities, there is a hint that eclipses may be deepest, on average, for low-metallicity stars. This possible trend is as predicted - planets orbiting low-metallicity hosts should have the lowest solid/gas ratios, making them the least dense and therefore biggest of planets.

Given that the running median is noisy and the slope of the local polynomial regression is small, is the trend statistically significant? The Kendall rank coefficient for mapping $[\mathrm{M} / \mathrm{H}]$ onto $R_{p} / R_{*}$ is $\tau=-0.108$. The associated probability that the null hypothesis - that $R_{p} / R_{*}$ is unrelated to star metallicity - is correct is $p=0.019$, a significance level at which the $\mathrm{R}$ statistical suite recommends adoption of the alternative hypothesis. For more information on Kendall's $\tau$ coefficient, see Abdi (2007). Using the large-sample Gaussian approximation to the variance $\nu$ of the $\tau$ sampling distribution,

$$
\nu=\frac{2(2 n+5)}{9 n(n-1)},
$$

where $n=213$ is the number of stars in the sample (Abdi 2007), I find that the standard deviation of the $\tau$ sampling distribution is 0.046 . The computed value of $\tau=-0.108$ differs from the $\tau=0$ expected in the case of the null hypothesis by $-2.3 \sigma$. I therefore consider the eclipse depth-metallicity trend interesting and suggestive, but not conclusive - especially since so little is known about the true distribution of giant planet eclipse depths that the Gaussian approximation to the $\tau$ distribution may not be appropriate. My main goal in writing this article is to motivate spectroscopic follow-up of the Kepler giant planet candidates. More precise metallicities and star radii will place our understanding of how planet structure evolves with star metallicity on much firmer statistical footing.

Besides the robust measures of center polotted in Figure 1 and the non-parametric Kendall's $\tau$ coefficient discussed above, there is another way to visualize the eclipse depth-metallicity trend. In Figure 2, I have divided the data into four broad metallicity bins and plotted the fraction of planet candidates for which $R_{p} / R_{*}>0.13$ in each bin. I chose $R_{p} / R_{*}=0.13$ as the cutoff because it is a roughly typical eclipse depth of well-studied inflated planets such as HD 209458 b $\left(R_{p} / R_{*}=0.12\right.$; Charbonneau et al. 2000) and HAT-P-19 b $\left(R_{p} / R_{*}=\right.$ 0.14; Hartman et al. 2011). What Figure 2 also hints at, then, is that stars in the lowest-metallicity bin tend to host a higher fraction of planets that are large in comparison to their host stars than the stars in the highermetallicity bins. Notice, however, that the Poisson error bars on the fraction of high eclipse depths in the lowestmetallicity bin overlap with the error bars on two of the other three bins. Furthermore, note that in binning the data and setting a threshold value of $R_{p} / R_{*}=0.13$, I have made the threshold and the bins parameters that affect any statistical inferences made from Figure 2 (though slightly different cutoff values yield a similar trend). Figure 2 is intended more as a visualization of the data than as a statistical tool.

There is one more potential pitfall left to examine, the accuracy of the $\mathrm{KIC}[\mathrm{M} / \mathrm{H}]$ estimates. Already the planet size-metallicity trend is on marginal footingcould $[\mathrm{M} / \mathrm{H}]$ inaccuracies wipe it out entirely? Figure 3 shows $\mathrm{KIC}[\mathrm{M} / \mathrm{H}]$ as a function of spectroscopically determined $[\mathrm{Fe} / \mathrm{H}]$ for confirmed, published Kepler discoveries. From Figure 3, one can see that the KIC metallicities are systematically lower than the spectroscopic values. The best-fit line relating $\mathrm{KIC}[\mathrm{M} / \mathrm{H}]$ to spectroscopic $[\mathrm{Fe} / \mathrm{H}]$ is

$$
[\mathrm{M} / \mathrm{H}]_{\mathrm{KIC}}=0.88[\mathrm{Fe} / \mathrm{H}]_{\mathrm{spec}}-0.14 \text {. }
$$

This result agrees with the analysis of Brown et al. (2011), whose comparison of KIC metallicities with $[\mathrm{Fe} / \mathrm{H}]$ derived from Keck/HIRES spectra uncovered a systematic 0.17-dex underestimate in the KIC values. The Spearman rank correlation coefficient relating $[\mathrm{Fe} / \mathrm{H}]_{\text {spec }}$ and $[\mathrm{M} / \mathrm{H}]_{\mathrm{KIC}}$ is 0.76 , which has a twosided significance of its deviation from zero of $2.7 \times 10^{-6}$. In contrast, Brown et al. (2011) found a Spearman rank correlation coefficient of only 0.42 , with a significance of 0.02 , for a subset of stars in the KIC observed with Keck/HIRES. My analysis indicates that, despite the differences in absolute scale between spectroscopic and $\mathrm{KIC}$ metallicities, the two are nevertheless closely related. Note, however, the two outliers, Kepler-10 and Kepler19. Kepler-10 is a Solar-radius, Solar-temperature star

1 Host star metallicities for Kepler discoveries were collected from the Extrasolar Planets Encyclopaedia, http://exoplanet.eu 
with G spectral type (Batalha et al. 2011), while Kepler19 is a slightly smaller star $\left(R_{*}=0.85 R_{\odot}\right.$, Ballard et al. 2011) with $T_{\text {eff }}=5541 \pm 60 \mathrm{~K}$ and a possibly later spectral type. Neither star falls into a known problem category for the $\mathrm{KIC}-\mathrm{F}$ stars, subgiants and stars with $T_{\text {eff }}<4200 \mathrm{~K}$ - so there is no clear reason why their KIC metallicities are so low. Published planet candidates used in the $[\mathrm{M} / \mathrm{H}]$ accuracy analysis are listed in Table 2 .

Knowing that KIC metallicities do track spectroscopic metallicities, albeit with some scatter, the relevant question for the purposes of this study is whether or not one can place the Kepler candidate planet hosts in the correct order on some metallicity scale. Keeping in mind the warnings about how binning introduces extra parameters into the statistical analysis whose effects may not be well understood, I still wish to know how often measurement error will cause a candidate planet host to cross over into a neighboring bin in Figure 2 The scatter of the $\mathrm{KIC}[\mathrm{M} / \mathrm{H}]$ values around the best-fit line in equation 2 is $\sigma_{[\mathrm{M} / \mathrm{H}]}=0.22 \mathrm{dex}$, indicated by dash-dotted lines in the left-hand panel of Figure 3. (The right-hand panel of Figure 3 shows $\mathrm{KIC}[\mathrm{M} / \mathrm{H}]$ as a function of $T_{\text {eff }}$, a correlation I will discuss further in 44.) I modeled the probability distribution of each star's true metallicity using a Gaussian distribution with $\sigma=0.22$ dex centered on that star's KIC $[\mathrm{M} / \mathrm{H}]$ value. These Gaussian distributions of probable metallicity formed the basis of a Monte Carlo simulation, in which I randomly sampled each star's $[\mathrm{M} / \mathrm{H}]$ distribution 20,000 times, divided the random samples into the same $[\mathrm{M} / \mathrm{H}]$ bins as in Figure 2 and counted the fraction of planet candidates in each bin with $R_{p} / R_{*}>0.13$.

Figure 5 shows the results of the Monte Carlo simulation. Here the story from the error bars is slightly more promising. Candidate planet hosts with $-0.85 \leq$ $[\mathrm{M} / \mathrm{H}] \leq-0.45$ dex show significantly more deep eclipses for which $R_{p} / R_{*}>0.13$ than stars with $[\mathrm{Fe} / \mathrm{H}]>$ -0.15 dex. Once again, though, the error bars barely miss overlapping, so that the statistical significance of the trend is about $2 \sigma$-suggestive, but not definitive. Confirming that gas giants orbiting low-metallicity stars really tend to have larger radii than gas giants orbiting high-metallicity stars will require extensive follow-up spectroscopy of the Kepler candidate planet hosts. The California-Kepler survey is already underway and will significantly improve stellar $\log (g),[\mathrm{Fe} / \mathrm{H}]$ and planet radius estimates (Howard et al. 2012). With spectroscopic observations of a large fraction of the giant planet candidates, it should even be possible to cast the sizemetallicity trend in terms of $R_{p}$ rather than $R_{p} / R_{*}$.

\section{STATISTICAL BIASES}

In this section I discuss possible statistical biases that could masquerade as a size-metallicity trend. The first possible bias I wish to examine is whether or not the $\mathrm{Ke}$ pler mission is equally sensitive to planets with minimum radius $5 R_{\oplus}$ in each metallicity bin. Given its ability to detect Earthlike planets transiting Sunlike stars, Kepler has at least 25 times the sensitivity required to detect planets with $R_{p}=5 R_{\oplus}$ orbiting Solar-type stars, which produce eclipse depths of two parts per thousand. Planets with $R_{p}=5 R_{\oplus}$ only become undetectable when the star radius approaches $5 R_{\odot}$. Since the largest star radius in the selected sample is $4.82 R_{\odot}$ and only five stars have $R_{*}>3 R_{\odot}$, there should be no systematic trend that prevents detection of the smaller, $5 R_{\oplus}$ giant planets at low metallicity.

Another possible bias arises from the interaction of two effects: the scarcity of gas giants orbiting low-mass stars (e.g. Bonfils et al. 2011) and the red colors of late K- and M-type stars. Among stars of a constant $J-H$ color, those that are most metal-rich tend to have the highest $g-r$ color because of the wealth of iron absorption lines in the blue part of the spectrum (Schlaufman \& Laughlin 2011). Red optical color is therefore an important metallicity indicator for stars in the KIC, but its usefulness breaks down for late-type stars that are naturally red, whatever their metallicity. Brown et al. (2011) show that the coolest stars, those with $T_{\text {eff }} \leq 4200 \mathrm{~K}$, tend to be artificially classified with super-Solar metallicity. Above $T_{\text {eff }}=4200 \mathrm{~K}$, the metallicity distribution is a well-behaved Gaussian that is not a function of temperature. One might worry that the low proportion of inflated giant planets orbiting the most metal-rich stars could be an artifact of the known scarcity of gas giants orbiting low-mass stars (Endl et al. 2006; Cumming et al. 2008; Bonfils et al. 2011). Perhaps low-mass stars whose metallicities are artificially high account for much of the apparently metal-rich part of the selected sample. Fortunately, only four stars in the sample have $T_{\text {eff }}<4200$, so the most problematic KIC metallicities have largely been avoided.

Although the analysis of KIC atmospheric parameters by Brown et al. (2011) does not show any obvious correlation between $\mathrm{KIC}[\mathrm{M} / \mathrm{H}]$ and $T_{\text {eff }}$ for stars with $T_{\text {eff }}>4200$ (see their Figure 11), I wish to independently verify the lack of a temperature-metallicity relationship. The right-hand panel of Figure 3 shows $[\mathrm{M} / \mathrm{H}]$ as a function of $T_{\text {eff }}$ for stars in the selected sample. The solid line indicates the mean $[\mathrm{M} / \mathrm{H}]$ value and the dash-dot line gives the best linear fit. The Spearman rank correlation coefficient is -0.19 , with a significance of 0.0049 three orders of magnitude higher than the significance of the $[\mathrm{M} / \mathrm{H}]_{\mathrm{KIC}}[\mathrm{Fe} / \mathrm{H}]_{\text {spec }}$ relationship (a high significance value indicates a weak correlation). While the correlation analysis shows some evidence for a metallicitytemperature relationship, KIC metallicity estimates appear to be much more related to true star metallicities than temperatures. It is likely that the eclipse depth$[\mathrm{M} / \mathrm{H}]$ correlation heralds a change in planet structure with host star $[\mathrm{M} / \mathrm{H}]$ rather than with host star mass and temperature, but I am unwilling to completely rule out the possibility that dearth of gas giants orbiting lowmass stars is affecting the results.

Although the Kepler planet radii used to select the sample show no correlation with $\log (g)$ (Figure 4 left panel), a possible degeneracy between $[\mathrm{M} / \mathrm{H}]$ and $\log (g)$ in the KIC could affect the results of this study. Red optical color is an indicator of both high $\log (g)$ and high metallicity, and the only intermediate-bandwidth filter used for KIC photometry - the D51 filter - is sensitive to both $\log (g)$ and $[\mathrm{M} / \mathrm{H}]$. Fortunately, the correlation between $\log (g)$ and $[\mathrm{M} / \mathrm{H}]$ is much weaker than the correlation between $T_{\text {eff }}$ and $[\mathrm{M} / \mathrm{H}]$. For $[\mathrm{M} / \mathrm{H}]$ as a function of $\log (g)$ (Figure 4, right panel), I find a Spearman rank correlation coefficient of -0.13 , with a significance of 0.05. Furthermore, the subgiant problem discussed 
earlier in this section should apply to a small fraction of the stars in the sample: only seven stars of 213 , or $3 \%$, have $\log (g)<0.7$. As with temperature, I am unwilling to definitively state that the weak degeneracy between $\mathrm{KIC} \log (g)$ and $[\mathrm{M} / \mathrm{H}]$ plays no part in creating the eclipse depth-metallicity correlation. However, I think it likely that declining eclipse depths with metallicity are related to giant planet interior structure rather than $\log (g)-[\mathrm{M} / \mathrm{H}]$ degeneracies.

The final possible source of bias comes from the possible 5\%-40\% contamination of the sample with eclipsing binaries, background eclipsing binaries, and hierarchical triples. This bias arises because stellar multiplicity rates depend on star mass. From $0.1 M_{\odot}-40 M_{\odot}$, the binary fraction is an increasing function of star mass (e.g. Clark et al. 2012), with about $20 \%$ of $0.1 M_{\odot}$ stars in binaries and $40 \%$ of $1 M_{\odot}$ stars in binaries. The increasing binary fraction with $M_{*}$ may mean that the planet candidates orbiting stars with the largest radii have the highest contamination rate. Since the tendency in the KIC is for low-mass, low-temperature stars to have artificially high metallicity values, it is possible for background eclipsing binaries to produce an eclipse depth-metallicity trend. However, the binary fraction is only a source of bias if $[\mathrm{M} / \mathrm{H}]$ is related to star temperature in the candidate giant planet sample. Given that my analysis indicates that KIC metallicity is more related to spectroscopic metallicity than any other stellar parameter, I do not think the multiple-star contamination is the source of the possible eclipse depth-metallicity trend-but, again, more followup is necessary to rule out multiple stars as a source of bias.

\section{PHYSICAL EXPLANATIONS FOR THE ECLIPSE DEPTH-METALLICITY CORRELATION}

Assuming that the tentative correlation between planet candidate eclipse depth and metallicity (1) is real, and (2) is not a manifestation of the dependence of planet occurrence rate on stellar mass - both of which are assumptions that require further investigation - what is the physical reason for the correlation? First, it is important to point out that for stars of a given mass and age, star radius decreases with increasing metallicity (e.g. Marigo et al. 2008) - so the fall in median $R_{p} / R_{*}$ with $[\mathrm{M} / \mathrm{H}]$ means gas giant interior structure must change substantially to counteract the fact that eclipse depth should tend to rise with star metallicity for a uniform population of planets. To the extent that the $\mathrm{KIC}[\mathrm{M} / \mathrm{H}]$ values track spectroscopic $[\mathrm{Fe} / \mathrm{H}]$, the eclipse depth-metallicity correlation reflects real changes in the population of giant planets as a function of star metallicity.

One possibility is that irradiation from the central star depends on metallicity. Irradiation provides the energy required to inflate hot Jupiters either through Ohmic dissipation (which depends on the star's ability to ionize the upper atmosphere; Batygin et al. 2011) or simply by forcing a shallow atmospheric temperature gradient that slows planetary contraction (e.g. Guillot \& Showman 2002; Baraffe et al. 2003; Burrows et al. 2003; Fortney \& Nettelmann 2011). At a given mass, a star of low metallicity will be slightly bluer and hotter than its counterpart at high metallicity, so metal-poor stars might tend to host larger-radius planets if the orbital distribution does not depend on metallicity. The points in Figure 1 are color-coded according to average stellar insolation. Although there is a visible tendency for least-irradiated planets to have the smallest eclipse depths (note the cluster of green points toward the bottom of the plot), there is no obvious tendency for the planets orbiting the lowest-metallicity hosts to be the most irradiated. I therefore tentatively rule out differences in irradiation levels as the reason for the eclipse depth-metallicity correlation.

Other planetary inflation mechanisms that have been proposed include tidal heating (Jackson et al. 2008), thermal tides (Arras \& Socrates 2010) and doublediffusive convection (Chabrier \& Baraffe 2007). Of these alternative inflation mechanisms, double-diffusive convection - in which composition gradients create multiple, semi-detached convective layers between which energy transport is inefficient - is the most likely to be related to star metallicity. In their models of the interior structures of uninflated hot Jupiters, Miller \& Fortney (2011) found a positive correlation between stellar metallicity and heavy-element mass in planetary envelopes (see their Figure 2). A metal-rich planetary envelope would likely have a steeper composition gradient than a metal-poor envelope. It is therefore possible that doublediffusive convection provides the required connection between giant planet structure and stellar metallicity.

The most straightforward explanation for the eclipse depth-metallicity trend, however, is that metal-rich planets of a given mass are denser than their metalpoor counterparts, leading to smaller radii (e.g. Fortney \& Nettelmann 2011). Transit searches have for some time provided hints that heavily metalenriched, dense planets tend to orbit metal-rich stars such as HD 149026 (Sato et al. 2005) and HAT-P-2 (Bakos et al. 2007; Leconte et al. 2009), and the connection between heavy-element mass and host star metallicity - the crucial ingredient behind any eclipse depth- $[\mathrm{M} / \mathrm{H}]$ correlation-has also been statistically established (Guillot et al. 2006; Miller \& Fortney 2011). Given that Jupiter, Saturn, Uranus and Neptune all orbit the same star but have radii ranging from $4.0 R_{\oplus}$ to $11.2 R_{\oplus}$, one expects to see gas giants with a range of radii orbiting stars of all metallicities yet probed (whether or not giant planets should form around Population II stars is an interesting question). However, if Jupiter, Saturn, Uranus and Neptune were all twice as metal-rich as they are, the average radius of the entire ensemble of Solar System planets would decline. I favor a simple density effect as the reason behind the increased eclipse depths of planet candidates orbiting low-metallicity stars.

I now make a speculative proposition, which is that low-metallicity stars may host a higher proportion of planets that formed by gravitational instability than high-metallicity stars. While dust is the necessary raw ingredient for the protoplanetary cores that nucleate the growth of massive gaseous atmospheres in the core accretion scenario, dust is the enemy of planet formation by gravitational instability because it increases disk opacity, reducing the disk's ability to cool (Cai et al. 2006). Though planets that form by core accretion tend to be metal-rich even relative to their host stars and by necessity have solid cores (Miller \& Fortney 2011), planets that form by gravitational instability most likely have 
the stellar composition and tend to be less dense (see, however, Helled \& Bodenheimer 2010). The idea that the giant planets orbiting low-metallicity stars predominantly formed by gravitational instability is interesting and warrants further thought. The theory that highmetallicity stars and low-metallicity stars form their gas giants by different mechanisms is consistent with the results of Santos et al. (2004) and Udry \& Santos (2007), who found that the frequency of giant planets as a function of $[\mathrm{Fe} / \mathrm{H}]$ is flat at subsolar metallicities. If the dominant planet formation mechanism were not changing with metallicity, one would expect a monotonically increasing planet detection rate with $[\mathrm{Fe} / \mathrm{H}]$ for the entire metallicity range surveyed.

While the eclipse depth-metallicity trend is not yet on firm statistical footing, it is an intriguing possibility that may provide insight into the Galactic history of planet formation. This work provides the first piece of evidence that giant planet structure, not just detection rate, depends on stellar metallicity. The planet populations of metal-rich stars and metal-poor stars may be very different. It is even possible that differences in planetary structure with host star metallicity could indicate not only changes in the raw ingredients available for planet formation as the Galaxy becomes chemically enriched, but changes in the planet formation mechanism itselfplanetary evolution across cosmic time.

Funding for this work was provided by the National Science Foundation through the Faculty Early Career Development (CAREER) program, award AST-1055910, to S. Dodson-Robinson. I thank Bill Cochran for input his extensive help with weeding out suspicious giant planet candidates from the Kepler database, Eric Feigelson for advice on nonparametric, robust statistical methods, and the anonymous referee for a thorough critique of the manuscript. This research has made use of the NASA Exoplanet Archive, the Exoplanet Encyclopedia and the SIMBAD database, operated at CDS, Strasbourg, France.

\section{REFERENCES}

Abdi, H. 2007, in Encyclopedia of Measurement and Statistics, ed. N. J. Salkind (Thousand Oaks, CA: Sage), 508

Arras, P., \& Socrates, A. 2010, ApJ, 714, 1

Bakos, G. Á., Kovács, G., Torres, G., et al. 2007, ApJ, 670, 826

Bakos, G. Á., Torres, G., Pál, A., et al. 2010, ApJ, 710, 1724

Ballard, S., Fabrycky, D., Fressin, F., et al. 2011, ApJ, 743, 200

Baraffe, I., Chabrier, G., Barman, T. S., Allard, F., \& Hauschildt, P. H. 2003, A\&A, 402, 701

Batalha, N. M., Borucki, W. J., Bryson, S. T., et al. 2011, ApJ, 729, 27

Batalha, N. M., Borucki, W. J., Koch, D. G., et al. 2010, ApJ, 713, L109

Batygin, K., Stevenson, D. J., \& Bodenheimer, P. 2011, ApJ, 738, 1

Bonfils, X., Delfosse, X., Udry, S., et al. 2011, A\&A, submitted (arXiv:1111.5019)

Bonomo, A. S., Hébrard, G., Santerne, A., Santos, N. C., Deleuil, M., Almenara, J., Bouchy, F., Díaz, R. F., Montou, C., \& Vanhuysse, M. 2011, arXiv:1110.5462

Borucki, W. J., Koch, D. G., Basri, G., et al. 2011, ApJ, 736, 19

Borucki, W. J., Koch, D. G., Batalha, N., et al. 2012, ApJ, 745, 120

Borucki, W. J., Koch, D. G., Brown, T. M., et al. 2010, ApJ, 713, L126

Bouchy, F., Bonomo, A. S., Santerne, A., et al. 2011, A\&A, 533, 83

Brown, T. M., Latham, D. W., Everett, M. E., \& Esquerdo, G. A. 2011, ApJ, 142, 112

Buchhave, L. A., Latham, D. W., Carter, J. A., et al. 2011, ApJS, 197, 3

Burrows, A., Sudarsky, D., \& Hubbard, W. B. 2003, ApJ, 594, 545

Cai, K., Durisen, R. H., Michael, S., Boley, A. C., Mejía, A. C., Pickett, M. K., \& D'Alessio, P. 2006, ApJ, 636, 149

Casagrande, L., Ramírez, I., Meléndez, J., Bessell, M., \& Asplund, M. 2010, A\&A, 512, 54

Chabrier, G., \& Baraffe, I. 2007, ApJ, 661, L81

Chabrier, G., Baraffe, I., Leconte, J., Gallardo, J., \& Barman, T. 2009, American Institute of Physics Conference Series, 1094, 102

Chaplin, W. J., Appourchaux, T., Elsworth, Y., et al. 2010, ApJ, 713, L169

Charbonneau, D., Brown, T. M., Latham, D. W., \& Mayor, M. 2000, ApJ, 529, L45

Clark, B. M., Blake, C. H., \& Knapp, G. R. 2012, ApJ, 744, 119

Cleveland, W. S., Grosse, E., \& Shyu, M. 1992, in Statistical Models in S, ed. J. Chambers \& T. Hastie (London: Chapman \& Hall), 309

Cochran, W. D., Fabrycky, D. C., Torres, G., et al. 2011, ApJS, 197,7
Cumming, A., Butler, R. P., Marcy, G. W., Vogt, S. S., Wright, J. T., \& Fischer, D. A. 2008, PASP, 120, 531

Demarcus, W. C. 1958, AJ, 62, 3

Doyle, L. R., Carter, J. A., Fabrycky, D. C., et al. 2011, Science, 333,1602

Endl, M., Cochran, W. D., Kürster, M., Paulson, D. B.

Wittenmyer, R. A., MacQueen, P. J., \& Tull, R. G. 2006, ApJ, 649, 436

Endl, M., MacQueen, P. J., Cochran, W. D., et al. 2011, ApJS, 197, 13

Fabrycky, D. C., Ford, E. B., Steffen, J. H., et al. 2012, arXiv: 1201.5415

Fischer, D. A., Schwamb, M. E., Schawinski, K., et al. 2012, MNRAS, 419, 2900

Fischer, D. A., \& Valenti, J. 2005, ApJ, 622, 1102

Ford, E. B., Fabrycky, D. C., Steffen, J. H., et al. 2012, arXiv: 1201.5409

Fortney, J. J., Demory, B.-O., Désert, J.-M., et al. 2011, ApJS, 197, 9

Fortney, J. J., \& Nettelmann, N. 2010, Space Science Reviews, 152,423

Fressin, F., Torres, G., Rowe, J. F., et al. 2011, Nature, online (doi:10.1038/nature10780)

Gillon, M., Demory, B.-O., Barman, T., Bonfils, X., Mazeh, T., Pont, F., Udry, S., Mayor, M., \& Queloz, D. 2007, A\&A, 471, L51

Gillon, M., Pont, F., Demory, B.-O., Mallmann, F., Mayor, M., Mazeh, T., Queloz, D., Shporer, A., Udry, S., \& Vuissoz, C. 2007, A\&A, 472, 13

Gonzalez, G. 1998, A\&A, 334, 221

Guillot, T., \& Showman, A. P. 2002, A\&A, 385, 156

Guillot, T., Santos, N. C., Pont, F., Iro, N., Melo, C., \& Ribas, I. 2006, A\&A, 423, L21

Härdle, W., \& Steiger, W. 1995, Applied Statistics, 44, 258

Hartman, J. D., Bakos, G. Á., Sato, B., et al. 2011, ApJ, 726, 52

Helled, R., Anderson, J. D., Podolak, M., \& Schubert, G. 2011, ApJ, 726, 15

Helled, R., \& Bodenheimer, P. 2010, Icarus, 207, 503

Holman, M. J., Fabrycky, D. C., Ragozzine, D., et al. 2010, Science, 330, 51

Howard, A., Marcy, G. W., Johnson, J. A., Morton, T. D., \& Isaacson, H. 2012, American Astronomical Society Meeting Abstracts 219, \#405.01

Jackson, B., Greenberg, R., \& Barnes, R. 2008, ApJ, 681, 1631

Jenkins, J. M., Borucki, W. J., Koch, D. G., et al. 2010, ApJ, 724,1108

Johnson, J. A., Gazak, J. Z., Apps, K., et al. 2011, arXiv: 1112.0017

Latham, D., Borucki, W., Koch, D., et al. 2010, ApJ, 713, L140 


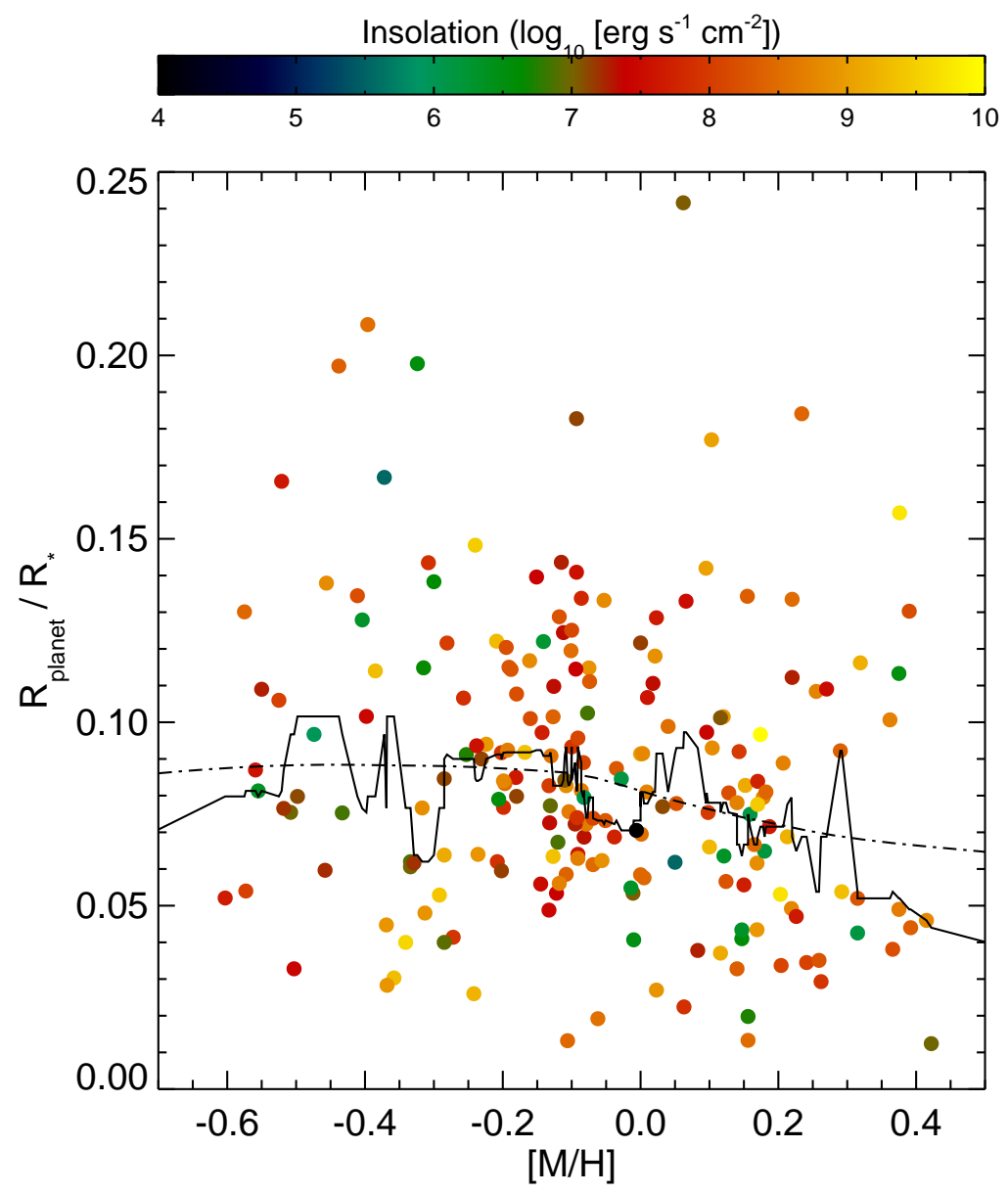

FIG. 1. - An analysis of the depth of Kepler planet candidate eclipses provides provides tentative support for the idea that planetary interior structure evolves with cosmic time. Here I plot $R_{p} / R_{*}$ as a function of host star $[\mathrm{M} / \mathrm{H}]$. The solid black line is a running median with a 21-point window, while the dash-dot line is a local polynomial regression of span 0.75 and degree 1 . Both robust measures of center show a decreasing trend in eclipse depth with $[\mathrm{M} / \mathrm{H}]$. Points on each plot are color-coded by level of stellar irradiation ("insolation") at the planet orbit. There is no indication that planets the orbiting low-metallicity hosts are more heavily irradiated than other planets.

Leconte, J., Baraffe, I., Chabrier, G., Barman, T., \& Levrard, B. 2009, A\&A, 506, 385

Lissauer, J. J., Fabrycky, D. C., Ford, E. B., et al. 2011, Nature, 470,53

Marigo, P., Girardi, L., Bressan, A., Groenewegen, M. A. T., Silva, L., \& Granato, G. L. 2008, A\&A, 482, 883

Miller, N., \& Fortney, J. J. 2011, ApJ, 736, L29

Morton, T. D., \& Johnson, J. A. 2011, ApJ, 738, 170

Nordström, B., Mayor, M., Andersen, J., Holmberg, J., Pont, F., Jørgensen, B. R., Olsen, E. H., Udry, S., \& Mowlavi, N. 2004, A\&A, 418, 989

Pinsonneault, M. H., An, D., Molenda-Żakowicz, J., Chaplin, W. J., Metcalfe, T. S., \& Bruntt, H. 2012, ApJS, 199, 30

Rogers, L. A., \& Seager, S. 2010, ApJ, 712, 974
Santerne, A., Bonomo, A. S., Hébrard, G., Deleuil, M., Moutou, C., Almenara, J.-M., Bouchy, F., \& Díaz, R. F. 2011, A\&A, 536,70

Santos, N. C., Israelian, G., \& Mayor, M. 2004, A\&A, 415, 1153

Schlaufman, K. C., \& Laughlin, G. 2011, ApJ, 738, 177

Skrutskie, M. F., Cutri, R. M., Stiening, R, et al. 2006, AJ, 131, 1163

Steffen, J. H., Fabrycky, D. C., Ford, E. B., et al. 2012, arXiv: 1201.5412

Sato, B., Fischer, D. A., Henry, G. W., et al. 2005, ApJ, 633, 465 Torres, G. 2007, ApJ, 671, L65

Torres, G., Winn, J. N., \& Holman, M. J. 2008, ApJ, 677, 1324

Udry, S., \& Santos, N. C. 2007, Annual Review of Astronomy and Astrophysics, 45, 397

Welsh, W. F., Orosz, J. A., Carter, J. A., et al. 2012, Nature, online (doi:10.1038/nature10768) 


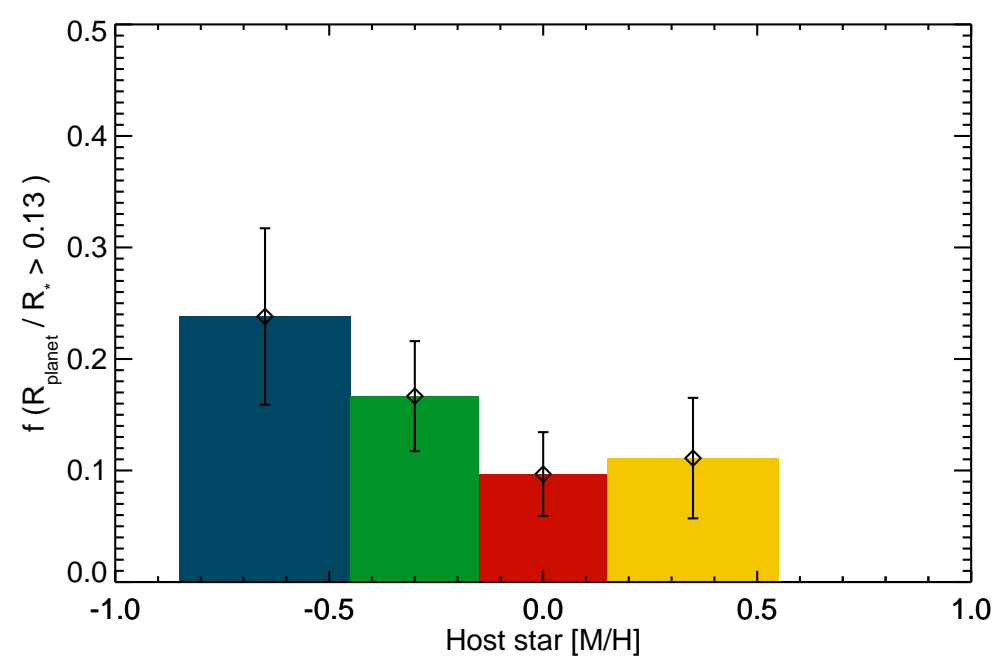

FIG. 2.- Fraction of giant planet candidates with $R_{p} / R_{*}>0.13$. Here the planets orbiting the lowest-metallicity hosts appear to be significantly bigger, relative to their parent stars, than the planets orbiting Solar-metallicity hosts.
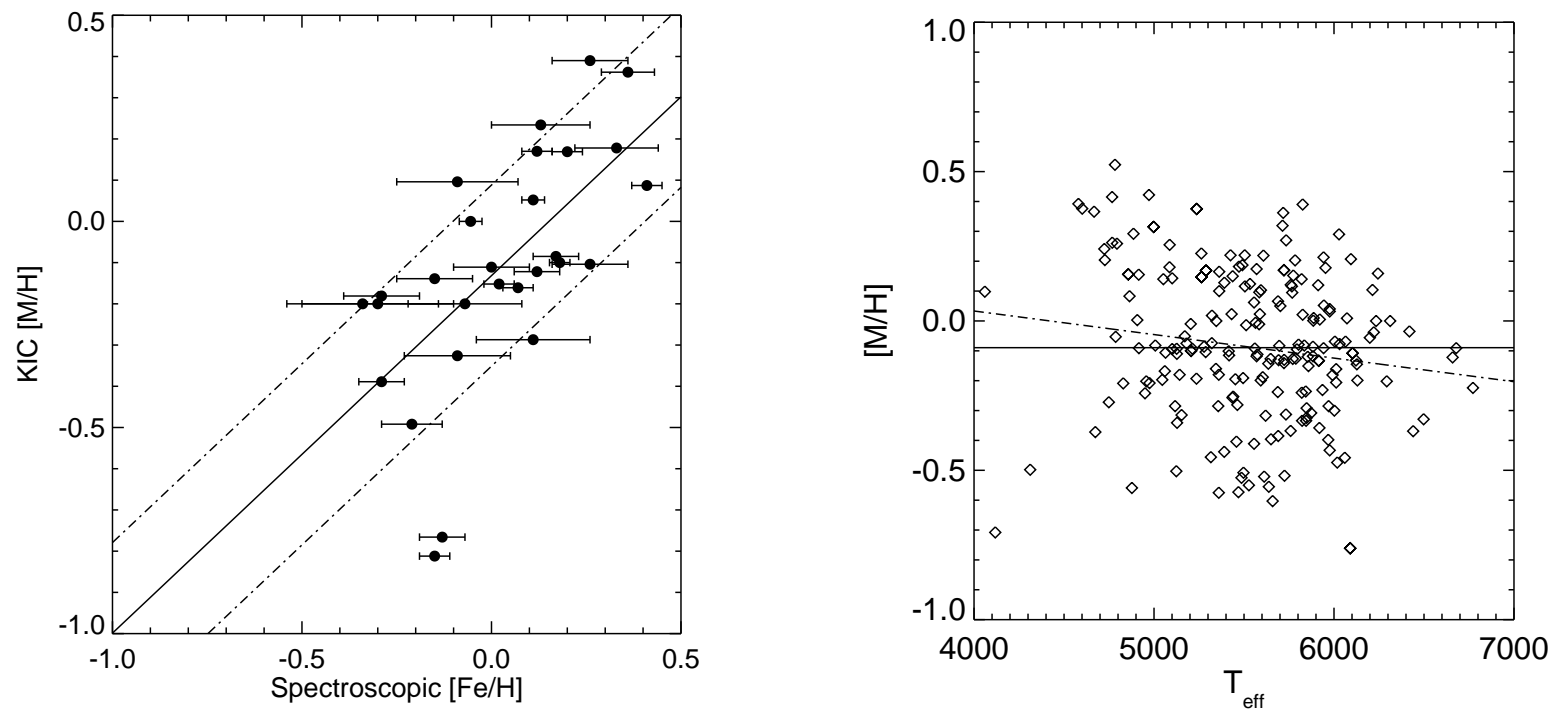

Fig. 3.- Left: A plot of $[\mathrm{M} / \mathrm{H}]$ from the Kepler Input Catalog (KIC) as a function of spectroscopially determined [M/H] for published Kepler discoveries provides insight into the precision and accuracy of KIC metallicities. The best-fit line (black) is $y=0.88 x-0.14$, indicating that KIC metallicities are systematically lower than their spectroscopic counterparts. The precision of the KIC metallicities, $\sigma_{[\mathrm{M} / \mathrm{H}]}=0.22$, is indicated by the dash-dotted lines. The two outliers, Kepler-10 and Kepler-19, are both Solar-type, single dwarf stars. Right: Here I investigate whether a $[\mathrm{M} / \mathrm{H}]$ is a function of $T_{\text {eff }}$ in the selected sample, a relationship that could introduce statistical biases due to changes in both planet and companion star occurrence rates as a function of stellar mass. The solid line shows the mean [M/H] of the sample, while the dash-dotted line shows the best-fit line representing $[\mathrm{M} / \mathrm{H}]$ vs. $T_{\text {eff }}$. The Spearman rank correlation coefficient is -0.19 , indicating a weak dependence of metallicity on temperature. 

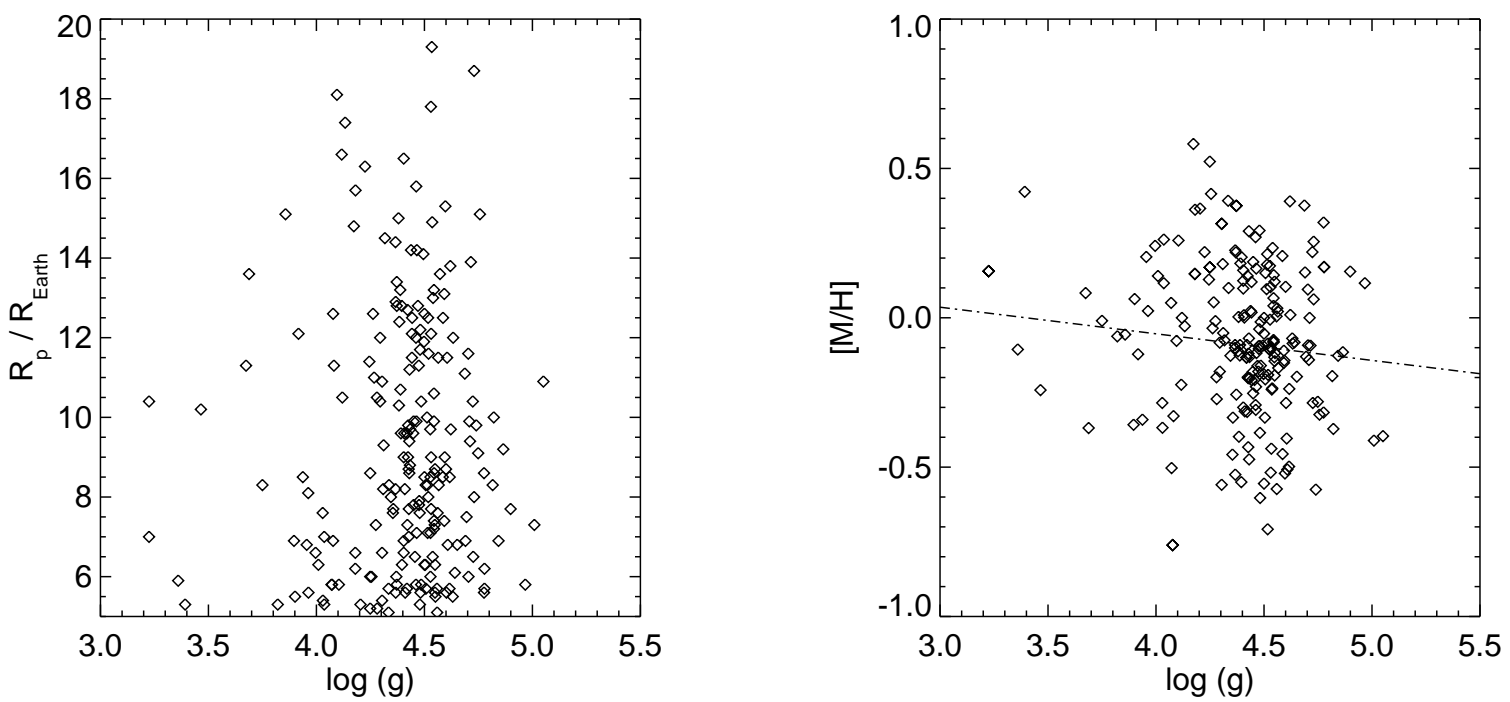

FIG. 4.- Left: The Spearman rank correlation coefficient for planet radius as a function of $\log (g)$ is 0.04 , with significance 0.55 , indicating no trend that could influence sample selection. Right: $[\mathrm{M} / \mathrm{H}]$ and $\log (g)$ values in the KIC are weakly correlated, with a Spearman rank coefficient of -0.13 . However, my analysis indicates that $\mathrm{KIC}[\mathrm{M} / \mathrm{H}]$ is more strongly related to spectroscopic $[\mathrm{Fe} / \mathrm{H}]$ than any other parameter. The dash-dot line shows the best fit representing $[\mathrm{M} / \mathrm{H}]$ as a function of $\log (g)$.

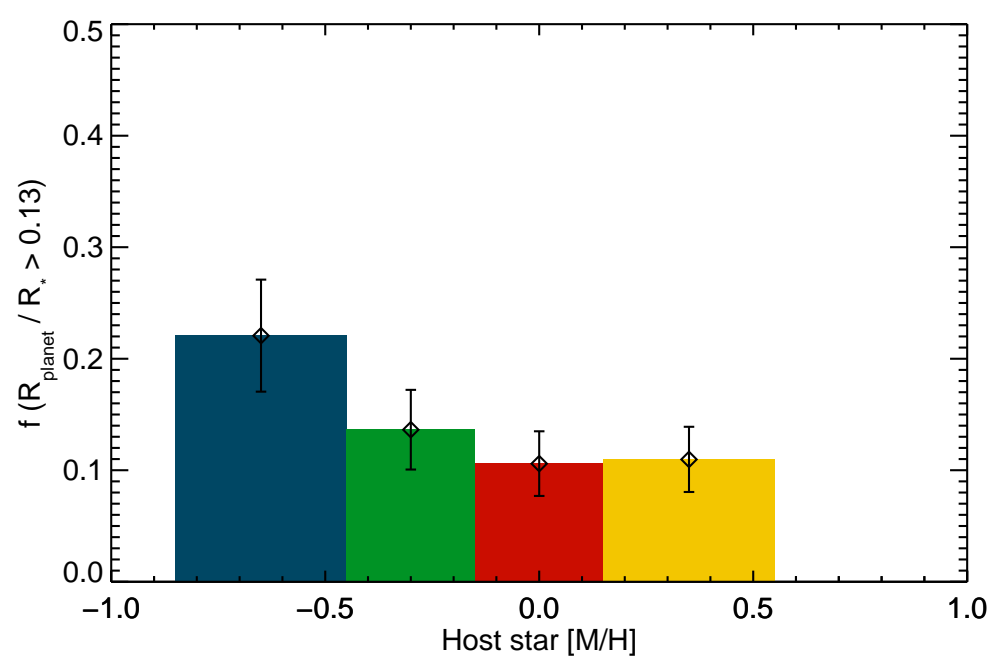

FIG. 5.- Monte Carlo simulations that take into account the errors in KIC metallicities suggest that there is a relationship between gas giant size and host star $[\mathrm{M} / \mathrm{H}]$. Here I show the fraction of systems with $R_{p} / R_{*}>0.13$ as a function of host star [Fe/H] from $2 \times 10^{4}$ Monte Carlo simulations, assuming errors on KIC metallicities are Gaussian and $\sigma_{[\mathrm{M} / \mathrm{H}]}=0.22$. The higher occurrence rate of large planets around the lowest-metallicity hosts, compared with Solar-metallicity and supersolar hosts, appears to be statistically significant. 
Dodson-Robinson

TABLE 1 Giant planet candidates used in analysis

\begin{tabular}{|c|c|c|c|c|}
\hline KOI number $^{\perp}$ & KIC number & $R_{p} / R_{*}$ & $\overline{R_{p} / R_{*} \text { error }}$ & $\overline{\mathrm{KIC}[\mathrm{M} / \mathrm{H}]}$ \\
\hline K00005.01 & 8554498 & 0.037 & 0.0002 & 0.12 \\
\hline K00010.01 & 6922244 & 0.091 & 0.0007 & 0.00 \\
\hline K00012.01 & 5812701 & 0.087 & 0.0001 & -0.04 \\
\hline K00020.01 & 11804465 & 0.117 & 0.0000 & -0.16 \\
\hline K00022.01 & 9631995 & 0.092 & 0.0001 & 0.29 \\
\hline K00063.01 & 11554435 & 0.057 & 0.0010 & 0.12 \\
\hline K00064.01 & 7051180 & 0.040 & 0.0003 & -0.34 \\
\hline K00082.01 & 10187017 & 0.034 & 0.0025 & 0.20 \\
\hline K00089.02 & 8056665 & 0.022 & 0.0006 & 0.06 \\
\hline K00094.01 & 6462863 & 0.070 & 0.0024 & -0.76 \\
\hline K00094.03 & 6462863 & 0.038 & 0.0016 & -0.76 \\
\hline K00097.01 & 5780885 & 0.078 & 0.0001 & 0.05 \\
\hline K00098.01 & 10264660 & 0.053 & 0.0094 & -0.12 \\
\hline K00100.01 & 4055765 & 0.045 & 0.0005 & -0.37 \\
\hline K00102.01 & 8456679 & 0.030 & 0.0024 & -0.36 \\
\hline K00105.01 & 8711794 & 0.039 & 0.0006 & -1.08 \\
\hline K00113.01 & 2306756 & 0.066 & 0.0210 & 0.10 \\
\hline K00127.01 & 8359498 & 0.097 & 0.0001 & 0.17 \\
\hline K00128.01 & 11359879 & 0.101 & 0.0005 & 0.36 \\
\hline K00131.01 & 7778437 & 0.075 & 0.0001 & 0.16 \\
\hline K00135.01 & 9818381 & 0.080 & 0.0074 & 0.18 \\
\hline K00137.01 & 8644288 & 0.043 & 0.0002 & 0.17 \\
\hline K00137.02 & 8644288 & 0.062 & 0.0013 & 0.17 \\
\hline K00138.01 & 8506766 & 0.094 & 0.0006 & -0.22 \\
\hline K00139.01 & 8559644 & 0.058 & 0.0022 & 0.01 \\
\hline K00144.01 & 4180280 & 0.035 & 0.0047 & 0.24 \\
\hline K00161.01 & 5084942 & 0.029 & 0.0068 & 0.26 \\
\hline K00183.01 & 9651668 & 0.122 & 0.0190 & -0.14 \\
\hline K00186.01 & 12019440 & 0.118 & 0.0001 & 0.02 \\
\hline K00187.01 & 7023960 & 0.142 & 0.0130 & 0.10 \\
\hline K00188.01 & 5357901 & 0.108 & 0.0001 & 0.26 \\
\hline K00189.01 & 11391018 & 0.133 & 0.0008 & -0.05 \\
\hline K00190.01 & 5771719 & 0.112 & 0.0006 & 0.22 \\
\hline K00191.01 & 5972334 & 0.115 & 0.0011 & -0.19 \\
\hline K00192.01 & 7950644 & 0.090 & 0.0210 & -0.23 \\
\hline K00193.01 & 10799735 & 0.129 & 0.0002 & -0.12 \\
\hline K00194.01 & 10904857 & 0.134 & 0.0037 & -0.09 \\
\hline K00195.01 & 11502867 & 0.114 & 0.0019 & -0.19 \\
\hline K00196.01 & 9410930 & 0.097 & 0.0009 & 0.10 \\
\hline K00197.01 & 2987027 & 0.091 & 0.0001 & 0.00 \\
\hline K00199.01 & 10019708 & 0.093 & 0.0170 & 0.10 \\
\hline K00200.01 & 6046540 & 0.083 & 0.0001 & 0.15 \\
\hline K00201.01 & 6849046 & 0.072 & 0.0001 & 0.19 \\
\hline K00202.01 & 7877496 & 0.102 & 0.0005 & 0.12 \\
\hline K00203.01 & 10619192 & 0.130 & 0.0002 & 0.39 \\
\hline K00204.01 & 9305831 & 0.076 & 0.0001 & -0.10 \\
\hline K00205.01 & 7046804 & 0.092 & 0.0020 & -0.17 \\
\hline K00206.01 & 5728139 & 0.063 & 0.0001 & -0.13 \\
\hline K00208.01 & 3762468 & 0.089 & 0.0007 & 0.21 \\
\hline K00209.01 & 10723750 & 0.069 & 0.0002 & -0.04 \\
\hline K00211.01 & 10656508 & 0.081 & 0.0960 & 0.01 \\
\hline K00212.01 & 6300348 & 0.064 & 0.0140 & -0.24 \\
\hline K00214.01 & 11046458 & 0.111 & 0.0035 & 0.02 \\
\hline K00216.01 & 6152974 & 0.065 & 0.0004 & 0.18 \\
\hline K00217.01 & 9595827 & 0.134 & 0.0001 & 0.22 \\
\hline K00229.01 & 3847907 & 0.049 & 0.0002 & 0.22 \\
\hline K00242.01 & 3642741 & 0.056 & 0.0002 & 0.15 \\
\hline K00254.01 & 5794240 & 0.184 & 0.0012 & 0.23 \\
\hline K00256.01 & 11548140 & 0.123 & 0.0023 & 0.58 \\
\hline K00261.01 & 5383248 & 0.027 & 0.0067 & 0.02 \\
\hline K00345.01 & 11074541 & 0.035 & 0.0063 & 0.26 \\
\hline K00348.01 & 11194032 & 0.038 & 0.0003 & 0.37 \\
\hline K00351.01 & 11442793 & 0.083 & 0.0064 & -0.11 \\
\hline K00351.02 & 11442793 & 0.059 & 0.0006 & -0.11 \\
\hline K00353.01 & 11566064 & 0.064 & 0.0012 & -0.09 \\
\hline K00356.01 & 11624249 & 0.033 & 0.0071 & -0.50 \\
\hline K00367.01 & 4815520 & 0.044 & 0.0085 & -1.53 \\
\hline K00368.01 & 6603043 & 0.085 & 0.0001 & -0.03 \\
\hline K00372.01 & 6471021 & 0.081 & 0.0093 & -0.56 \\
\hline K00375.01 & 12356617 & 0.077 & $\ldots$ & -0.13 \\
\hline K00377.01 & 3323887 & 0.078 & 0.0030 & 0.17 \\
\hline K00377.02 & 3323887 & 0.084 & 0.0017 & 0.17 \\
\hline K00398.01 & 9946525 & 0.092 & 0.0036 & 0.14 \\
\hline K00401.01 & 3217264 & 0.041 & 0.0003 & 0.15 \\
\hline K00401.02 & 3217264 & 0.043 & 0.0034 & 0.15 \\
\hline K00410.01 & 5449777 & 0.102 & 0.0050 & -0.40 \\
\hline K00412.01 & 5683743 & 0.053 & 0.0002 & -0.01 \\
\hline
\end{tabular}


Eclipse depth-metallicity correlation

TABLE 1 Giant planet candidates used in analysis

\begin{tabular}{|c|c|c|c|c|}
\hline KOI number $^{1}$ & KIC number & $R_{p} / R_{*}$ & $R_{p} / R_{*}$ error & $\mathrm{KIC}[\mathrm{M} / \mathrm{H}]$ \\
\hline K00415.01 & 6289650 & 0.062 & 0.0630 & -0.33 \\
\hline K00417.01 & 6879865 & 0.097 & 0.0017 & -0.14 \\
\hline K00418.01 & 7975727 & 0.115 & 0.0004 & -0.32 \\
\hline K00419.01 & 8219673 & 0.091 & 0.0004 & -0.13 \\
\hline K00421.01 & 9115800 & 0.115 & 0.0002 & -0.07 \\
\hline K00422.01 & 9214713 & 0.138 & $\ldots$ & -0.30 \\
\hline K00423.01 & 9478990 & 0.085 & 0.0003 & -0.18 \\
\hline K00425.01 & 9967884 & 0.133 & 0.0140 & 0.07 \\
\hline K00428.01 & 10418224 & 0.056 & 0.0001 & -0.14 \\
\hline K00433.01 & 10937029 & 0.049 & 0.0180 & 0.38 \\
\hline K00433.02 & 10937029 & 0.113 & 0.0013 & 0.38 \\
\hline K00458.01 & 7504328 & 0.077 & 0.0062 & -0.20 \\
\hline K00464.01 & 8890783 & 0.067 & 0.0003 & 0.17 \\
\hline K00469.01 & 9703198 & 0.061 & 0.0029 & -0.07 \\
\hline K00523.01 & 8806123 & 0.063 & 0.0015 & -0.09 \\
\hline K00552.01 & 5122112 & 0.097 & 0.0013 & -0.47 \\
\hline K00554.01 & 5443837 & 0.069 & 0.0034 & -0.08 \\
\hline K00607.01 & 5441980 & 0.075 & 0.0009 & -0.51 \\
\hline K00609.01 & 5608566 & 0.089 & 0.0110 & -0.08 \\
\hline K00611.01 & 6309763 & 0.073 & 0.0004 & -0.13 \\
\hline K00617.01 & 9846086 & 0.177 & 0.0210 & 0.10 \\
\hline K00620.01 & 11773022 & 0.072 & 0.0009 & -0.08 \\
\hline K00622.01 & 12417486 & 0.073 & 0.0025 & -0.05 \\
\hline K00625.01 & 4449034 & 0.062 & 0.0097 & -0.06 \\
\hline K00633.01 & 4841374 & 0.028 & 0.0023 & -0.37 \\
\hline K00674.01 & 7277317 & 0.038 & 0.0042 & 0.08 \\
\hline K00680.01 & 7529266 & 0.060 & 0.0001 & -0.46 \\
\hline K00684.01 & 7730747 & 0.041 & 0.0042 & -0.01 \\
\hline K00686.01 & 7906882 & 0.108 & 0.0032 & -0.18 \\
\hline K00716.01 & 9846348 & 0.061 & 0.0027 & -0.33 \\
\hline K00725.01 & 10068383 & 0.083 & 0.0024 & -0.20 \\
\hline K00728.01 & 10221013 & 0.099 & 0.0010 & 0.04 \\
\hline K00737.01 & 10345478 & 0.064 & 0.0020 & -0.28 \\
\hline K00741.01 & 10418797 & 0.242 & 0.0062 & 0.06 \\
\hline K00743.01 & 10464078 & 0.087 & 0.0210 & -0.56 \\
\hline K00745.01 & 10485250 & 0.092 & 0.0006 & -0.20 \\
\hline K00753.01 & 10811496 & 0.102 & 0.0072 & -0.13 \\
\hline K00760.01 & 11138155 & 0.107 & 0.0008 & 0.01 \\
\hline K00763.01 & 11242721 & 0.110 & 0.0009 & -0.13 \\
\hline K00764.01 & 11304958 & 0.047 & 0.0004 & 0.23 \\
\hline K00767.01 & 11414511 & 0.128 & 0.0008 & 0.02 \\
\hline K00771.01 & 11465813 & 0.124 & $\ldots$ & -0.11 \\
\hline K00772.01 & 11493732 & 0.070 & 0.0044 & 0.00 \\
\hline K00774.01 & 11656840 & 0.143 & 0.0004 & -0.31 \\
\hline K00779.01 & 11909839 & 0.109 & 0.0210 & -0.55 \\
\hline K00782.01 & 11960862 & 0.048 & 0.0930 & -0.31 \\
\hline K00791.01 & 12644822 & 0.071 & 0.0003 & -0.01 \\
\hline K00797.01 & 3115833 & 0.077 & 0.0007 & -0.52 \\
\hline K00801.01 & 3351888 & 0.081 & 0.0130 & 0.18 \\
\hline K00802.01 & 3453214 & 0.135 & 0.0049 & -0.41 \\
\hline K00805.01 & 3734868 & 0.119 & 0.0095 & -0.10 \\
\hline K00806.01 & 3832474 & 0.093 & 0.0073 & -0.10 \\
\hline K00806.02 & 3832474 & 0.125 & 0.0005 & -0.10 \\
\hline K00809.01 & 3935914 & 0.114 & 0.0650 & -0.39 \\
\hline K00813.01 & 4275191 & 0.085 & 0.0003 & -0.28 \\
\hline K00815.01 & 4544670 & 0.101 & 0.0130 & -0.16 \\
\hline K00822.01 & 5077629 & 0.128 & 0.0015 & -0.40 \\
\hline K00823.01 & 5115978 & 0.075 & 0.0015 & -0.43 \\
\hline K00824.01 & 5164255 & 0.122 & 0.0013 & -0.21 \\
\hline K00830.01 & 5358624 & 0.134 & 0.0001 & 0.15 \\
\hline K00838.01 & 5534814 & 0.072 & 0.0010 & -0.10 \\
\hline K00840.01 & 5651104 & 0.096 & 0.0031 & -0.09 \\
\hline K00843.01 & 5881688 & 0.053 & 0.0049 & 0.20 \\
\hline K00846.01 & 6061119 & 0.166 & 0.0008 & -0.52 \\
\hline K00847.01 & 6191521 & 0.054 & 0.0330 & -0.57 \\
\hline K00850.01 & 6291653 & 0.092 & 0.0042 & -0.19 \\
\hline K00851.01 & 6392727 & 0.056 & 0.0007 & -0.12 \\
\hline K00855.01 & 6522242 & 0.138 & 0.0002 & -0.46 \\
\hline K00856.01 & 6526710 & 0.140 & 0.0025 & -0.15 \\
\hline K00858.01 & 6599919 & 0.091 & 0.0027 & -0.25 \\
\hline K00865.01 & 6862328 & 0.074 & 0.0360 & -0.09 \\
\hline K00868.01 & 6867155 & 0.161 & 0.0025 & -0.71 \\
\hline K00871.01 & 7031517 & 0.208 & 0.0044 & -0.40 \\
\hline K00872.01 & 7109675 & 0.084 & 0.0027 & -0.11 \\
\hline K00876.01 & 7270230 & 0.144 & 0.0066 & -0.12 \\
\hline K00878.01 & 7303253 & 0.041 & 0.0031 & -0.27 \\
\hline K00880.02 & 7366258 & 0.055 & 0.0004 & -0.01 \\
\hline
\end{tabular}


TABLE 1 Giant planet candidates used in analysis

\begin{tabular}{|c|c|c|c|c|}
\hline KOI number ${ }^{1}$ & $\overline{\text { KIC number }}$ & $\bar{R} / R_{*}$ & $R_{p} / R_{*}$ error & KIC $[\mathrm{M} / \mathrm{H}]$ \\
\hline K00882.01 & 7377033 & 0.151 & 0.0015 & -1.24 \\
\hline K00883.01 & 7380537 & 0.167 & 0.0005 & -0.37 \\
\hline K00889.01 & 757450 & 0.114 & 0.0003 & -0.09 \\
\hline K00890.01 & 7585481 & 0.077 & 0.0003 & 0.03 \\
\hline K00895.01 & 7767559 & 0.107 & 0.0011 & -0.26 \\
\hline K00897.01 & 7849854 & 0.109 & 0.0001 & 0.27 \\
\hline K00902.01 & 8018547 & 0.080 & 0.0007 & -0.50 \\
\hline K00903.01 & 8039892 & 0.077 & 0.0002 & -0.32 \\
\hline K00908.01 & 8255887 & 0.081 & 0.0002 & 0.13 \\
\hline K00913.01 & 8544996 & 0.122 & 0.0049 & -0.28 \\
\hline K00918.01 & 8672910 & 0.111 & 0.0003 & -0.07 \\
\hline K00929.01 & 9141746 & 0.078 & 0.0002 & 0.14 \\
\hline K00931.01 & 9166862 & 0.116 & 0.0075 & 0.32 \\
\hline K00941.01 & 9480189 & 0.043 & 0.0005 & 0.32 \\
\hline K00941.03 & 9480189 & 0.052 & 0.0120 & 0.32 \\
\hline K00951.01 & 9775938 & 0.046 & 0.0120 & 0.41 \\
\hline K00956.01 & 9875711 & 0.044 & 0.0170 & 0.39 \\
\hline K00960.01 & 8176650 & 0.183 & 0.0005 & -0.09 \\
\hline K00972.01 & 11013201 & 0.019 & 0.0025 & -0.06 \\
\hline K00981.01 & 8607720 & 0.013 & 0.0012 & -0.11 \\
\hline K00988.01 & 2302548 & 0.033 & 0.0021 & 0.14 \\
\hline K01003.01 & 2438502 & 0.141 & 0.0007 & -0.09 \\
\hline K01005.01 & 5780460 & 0.062 & 0.0430 & -0.21 \\
\hline K01089.01 & 3247268 & 0.083 & 0.0003 & -0.13 \\
\hline K01089.02 & 3247268 & 0.049 & 0.0060 & -0.13 \\
\hline K01159.01 & 10354039 & 0.054 & 0.0039 & 0.29 \\
\hline K01176.01 & 3749365 & 0.157 & 0.0003 & 0.38 \\
\hline K01177.01 & 3547091 & 0.130 & 0.0030 & -0.57 \\
\hline K01193.01 & 3942446 & 0.106 & 0.0380 & -0.53 \\
\hline K01208.01 & 3962440 & 0.060 & $\ldots$ & -0.20 \\
\hline K01221.02 & 3640905 & 0.012 & 0.0059 & 0.42 \\
\hline K01227.01 & 6629332 & 0.120 & 0.0013 & -0.20 \\
\hline K01241.01 & 6448890 & 0.020 & 0.0039 & 0.16 \\
\hline K01241.02 & 6448890 & 0.013 & 0.0051 & 0.16 \\
\hline K01242.01 & 6607447 & 0.058 & 0.0006 & 0.00 \\
\hline K01257.01 & 8751933 & 0.080 & 0.0005 & -0.18 \\
\hline K01261.01 & 8678594 & 0.064 & 0.0005 & 0.12 \\
\hline K01268.01 & 8813698 & 0.074 & & -0.07 \\
\hline K01285.01 & 10599397 & 0.081 & 0.0029 & -0.09 \\
\hline K01288.01 & 10790387 & 0.084 & 0.0005 & -0.20 \\
\hline K01299.01 & 10864656 & 0.026 & 0.0002 & -0.24 \\
\hline K01335.01 & 4155328 & 0.040 & 0.0210 & -0.28 \\
\hline K01353.01 & 7303287 & 0.102 & 0.0011 & -0.08 \\
\hline K01385.01 & 9278553 & 0.198 & 0.0003 & -0.32 \\
\hline K01391.01 & 8958035 & 0.079 & 0.0016 & -0.21 \\
\hline K01419.01 & 11125936 & 0.053 & 0.0035 & -0.29 \\
\hline K01426.02 & 11122894 & 0.067 & 0.0028 & -0.12 \\
\hline K01459.01 & 9761199 & 0.075 & 0.0010 & 0.10 \\
\hline K01474.01 & 12365184 & 0.062 & 0.0005 & -0.33 \\
\hline K01477.01 & 7811397 & 0.122 & $\ldots$ & 0.00 \\
\hline K01486.01 & 7898352 & 0.094 & & -0.24 \\
\hline K01540.01 & 5649956 & 0.197 & 0.0011 & -0.44 \\
\hline K01543.01 & 5270698 & 0.148 & 0.0003 & -0.24 \\
\hline K01546.01 & 5475431 & 0.101 & 0.0008 & 0.12 \\
\hline K01553.01 & 7951018 & 0.069 & 0.0006 & 0.21 \\
\hline K01557.01 & 5371776 & 0.039 & 0.0350 & 0.52 \\
\hline K01561.01 & 4940438 & 0.052 & 0.0016 & -0.60 \\
\hline K01574.01 & 10028792 & 0.062 & 0.0003 & 0.05 \\
\hline K01587.01 & 9932970 & 0.080 & 0.0067 & -0.08 \\
\hline
\end{tabular}

${ }^{1}$ Kepler Object of Interest number 
TABLE 2

Kepler DISCOVERIES USED FOR METALLICITY ACCURACY ANALYSIS

\begin{tabular}{|c|c|c|c|}
\hline Name & KIC number & Spectroscopic $[\mathrm{Fe} / \mathrm{H}]$ & Reference \\
\hline TrES-2 host star & 11446443 & $-0.15 \pm 0.1$ & Torres et al. (2008) \\
\hline Kepler-4 & 11853905 & $0.17 \pm 0.06$ & Borucki et al. (2010) \\
\hline Kepler-7 & 5780885 & $0.11 \pm 0.03$ & Latham et al. (2010) \\
\hline Kepler-8 & 6922244 & $-0.06 \pm 0.03$ & Jenkins et al. (2010) \\
\hline Kepler-9 & 3323887 & $0.12 \pm 0.04$ & Holman et al. (2010) \\
\hline Kepler-10 & 11904151 & $-0.15 \pm 0.04$ & Batalha et al. (2011) \\
\hline Kepler-11 & 6541920 & $0.00 \pm 0.10$ & Lissauer et al. (2011) \\
\hline Kepler-12 & 11804465 & $0.07 \pm 0.04$ & Fortney et al. (2011) \\
\hline Kepler-14 & 10264660 & $0.12 \pm 0.06$ & Buchhave et al. (2011) \\
\hline Kepler-15 & 11359879 & $0.36 \pm 0.07$ & Endl et al. (2011) \\
\hline Kepler-16AB & 12644769 & $-0.3 \pm 0.2$ & Doyle et al. (2011) \\
\hline Kepler-17 & 10619192 & $0.26 \pm 0.1$ & Bonomo et al. (2011) \\
\hline Kepler-18 & 8644288 & $0.2 \pm 0.04$ & Cochran et al. (2011) \\
\hline Kepler-19 & 2571238 & $-0.13 \pm 0.06$ & Ballard et al. (2011) \\
\hline Kepler-20 & 6850504 & $0.02 \pm 0.04$ & Fressin et al. \\
\hline Kepler-22 & 10593626 & $-0.29 \pm 0.06$ & Borucki et al. (2012b) \\
\hline Kepler-23 & 11512246 & $-0.09 \pm 0.14$ & Ford et al. (2012) \\
\hline Kepler-26 & 9757613 & $-0.21 \pm 0.08$ & Steffen et al. (2012) \\
\hline Kepler-27 & 5792202 & $0.41 \pm 0.04$ & Steffen et al. (2012) \\
\hline Kepler-30 & 3832474 & $0.18 \pm 0.027$ & Fabrycky et al. (2012) \\
\hline Kepler-34AB & 8572936 & $-0.07 \pm 0.15$ & Welsh et al. (2012) \\
\hline Kepler-35AB & 9837578 & $-0.34 \pm 0.2$ & Welsh et al. (2012) \\
\hline KOI-135 & 9818381 & $0.33 \pm 0.11$ & Bonomo et al. (2011) \\
\hline KOI-196 & 9410930 & $-0.09 \pm 0.16$ & Santerne et al. (2011) \\
\hline KOI-204 & 9305831 & $0.26 \pm 0.1$ & Bonomo et al. (2011) \\
\hline & 5794249 & $0.13 \pm 0.13$ & Johnson et al. (2011) \\
\hline \multirow{2}{*}{ KOI- 423} & 9478990 & $-0.29 \pm 0.1$ & Bouchy et al. (2011) \\
\hline & 6185331 & $0.11 \pm 0.15$ & Fischer et al. (2012) \\
\hline
\end{tabular}

